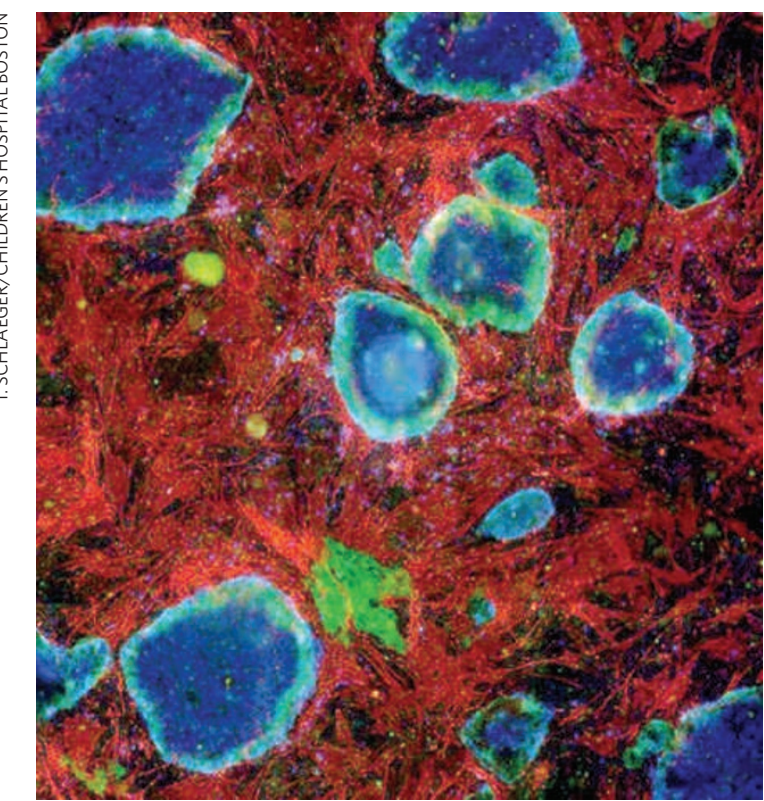

Human embryonic stem cells on mouse embryonic fibroblasts. help certain cell types but not others to survive. Both ideas would ultimately result in the same blood cells, but the path by which the cells got there would be radically different. As such, the two hypotheses would hold vastly different implications for treating diseases or generating blood in the laboratory.

To tease apart the actual mechanism, Schroeder's group took pictures of mouse blood cells every two to three minutes for several days. Because the researchers did not observe extensive cell death, their time-lapse film firmly supported the active instruction over the passive-survival hypothesis ${ }^{3}$. Long-term images make "a big, big difference", says Schroeder. "You can say, 'this is how it was', not 'this is how it probably was."

Researchers working in the fastpaced field of stem-cell reprogramto do." So researchers are planning ahead, and devising new systems for tracking cells in real time.

\section{Getting answers}

Beyond offering a lens onto new biology, long-term imaging studies are also beginning to resolve long-standing debates in developmental and cell biology. For example, Timm Schroeder, a stem-cell biologist at the Helmholtz Centre in Munich, Germany, led a team that used continuous imaging to distinguish between two competing hypotheses about the role of cytokines in blood development.

One view held by some immunologists is that cytokines - regulatory proteins found in the immune system - cause cells to take on new fates; another theory is that cytokines ming have also been keen to track how cells take on desired fates. Under most experimental set-ups, the early events are the hardest to follow. But a team led by George Daley and Thorsten Schlaeger, stem-cell biologists at the Children's Hospital Boston in Massachusetts, used long-term imaging to reveal the history of rare, reprogrammed cells.

To identify the presence of expected molecules on cell surfaces, the researchers added fluorescently tagged antibodies to the culture media as the cells grew into the colonies characteristic of induced pluripotent

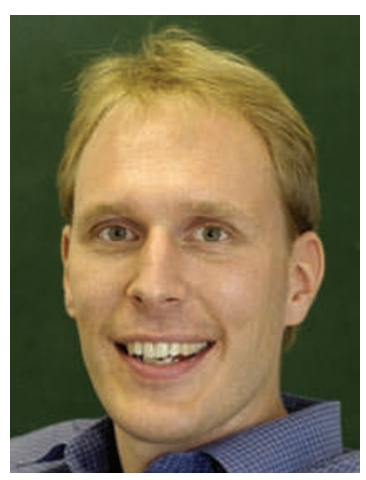

Timm Schroeder observes cells to disinguish between competing hypotheses. stem (iPS) cells. Meanwhile, they set their microscope to scan the cells constantly, making a complete survey of the 4-square-centimetre area every two or three days for about two weeks. After assessing which colonies produced high-quality iPS cells, the team could go back to the images to identify the cell clusters that gave rise to fully reprogrammed cells, even though each group of iPS cells took up as little as $0.0003 \%$ of the scanned area ${ }^{4}$.

Learning the hallmarks of iPS cells as they undergo reprogramming could not only yield better methods for growing patient-specific stem cells, but also prevent weeks of wasted effort (and costs) in animal experiments, says Schlaeger.

\section{Come to light}

These types of study are starting to shed light on hitherto unsolvable biological problems, such as why some patterns of cell division contribute to cancer and which progenitor cells give rise to blood, sperm, neurons or other tissue types. But researchers using livecell imaging have to be careful not to shed too much light - quite literally — because illuminating cells for long durations can damage cells or alter their behaviour. Schroeder's advice to biologists is to take the worst image possible to get the necessary data. "If you're pushing the envelope, you should aim for having healthy cells rather than the best images," he says. Beautiful images, he notes, often make for unhealthy cells.

So instead of continuous snapshots, researchers often rely on taking pictures at less frequent intervals. Many factors contribute to the imaging

\title{
A LONG-TERM LIVE-CELL COMMITMENT
}

The decision to undertake a long-term imaging project is not trivial. Experts suggest questions that researchers should ask themselves before starting out.

\section{How frequently do you need to} take an image?

Tracking individual cells often requires taking an image every few minutes. The more dense and mobile the cells are, the less time can elapse between images. For example, Michel Cayouette at the Clinical Research Institute of Montréal, Canada, takes images of retinal progenitor cells every seven minutes until they develop into neurons, at which stage he slows the rate of image acquisition to roughly once an hour.

\section{Can your cells survive the experiment?}

Repeated imaging can harm cells, especially when the imaging requires high-energy light. But the tolerance of different cells for fluorescence varies widely. Blood-forming stem cells are generally more robust than neural stem cells, for example, and thus can be imaged more frequently without affecting cell behaviour, notes Tannishtha Reya, a stem-cell biologist at Duke University in Durham, North Carolina.

\section{Can you keep calm?} Inexperienced researchers sometimes set up their longterm microscope systems in the middle of a heavily trafficked work station or worse, under ventilation systems. Such disturbances can easily overwhelm a system's ability to maintain stable conditions and can cause obfuscating artefacts, cautions Cayouette.

\section{Can you follow your cells?} Following cells in culture gets complicated once cells start crawling under and over each other. To track individual cells at low densities, labelling nuclei with Hoeschst often works well, says Thorsten Schlaeger at the Children's Hospital Boston in Massachusetts, although he cautions that some cells stain poorly, and non-toxic genetic labels can work better. If cells must be grown at high density, consider mixing in a few labelled cells and tracking just these.

\section{Are you computationally} prepared?

Crunching through large data sets can easily go beyond the capacity of standard lab computers, and a single experiment can completely fill a computer's hard drive.

Researchers need appropriate servers and back-up systems. A dedicated informatics set-up and the help of a programmer are "highly desirable", says Schlaeger. 